\title{
Craniotomy for suprasellar meningioma during pregnancy: role of fetal monitoring
}

\author{
Alison Macarthur MSc MD FRCPC
}

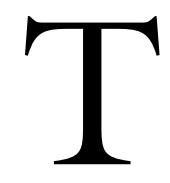

HE case report of a parturient with acuteonset neurological disease at 28 weeks gestation in this Canadian Journal of Anesthesia highlights the balancing act of treating mother and baby during non-obstetric disease. ${ }^{1}$ In this case, a woman with life-altering neurological symptoms from a suprasellar meningioma must receive urgent surgical treatment prior to her fetus reaching a reasonable gestational age for delivery. Non-obstetric surgery during pregnancy is not new and has been reported with surgical techniques, such as laparoscopic and cardiopulmonary bypass procedures. The risks of non-obstetric surgery include teratogenic consequences and onset of premature labour and premature delivery. The likelihood of premature labour and delivery following a neurosurgical procedure is less than with an intra-abdominal procedure, and the risk of teratogenicity has generally passed after the 12th week. However, a theoretical risk remains for intraoperative fetal asphyxia and whether we can detect this by intraoperative external fetal heart rate (FHR) monitoring. I look to the medical literature to help define the risk of asphyxia and to evaluate the utility of FHR monitoring.

If the fetus was at significant risk for intraoperative asphyxia, pregnancies following non-obstetric surgery would have increased rates of intra-uterine deaths (abortions, stillbirths), neonatal deaths, intra-uterine growth retardation or severe neurological deficits. To identify increased rates would require comparative information both on women who had non-obstetric surgery during pregnancy and on women who did not have surgery. Adverse outcomes, such as mortality or morbidity, are most efficiently evaluated with observational study designs, as it would be unethical to conduct the gold-standard a randomized clinical trial (randomizing pregnant women to receive surgical or placebo procedures). A cohort study would begin with the population of all pregnant women and com- pare the outcomes of women who have undergone non-obstetric surgery to women who have not required surgery. Case control studies, on the other hand, would begin by identifying women who had an adverse outcome (stillbirth or neonatal death) and compare them to women who did not have an adverse outcome, looking for potential risk factors such as non-obstetric surgical procedures.

A search for published cohort studies identified four investigators from Sweden, Canada and the U.S.A. who used population-based data of births outcomes. ${ }^{2-5}$ The four studies included approximately 2,000,000 Swedish pregnant women over 20 years, 100,000 pregnant Canadian women over seven years, and 70,000 pregnant American women over ten years. The incidence of non-obstetric surgery was reported between $0.2 \%$ and $0.79 \%$ of the pregnant population. The two Swedish studies compared stillbirth, neonatal death, and premature birth rates between women who had non-obstetric surgery and those who did not. ${ }^{2,3}$ Neither studies identified an increased risk for stillbirth following non-obstetric surgery, nor did the Canadian study identify an increased risk for abortion (intra-uterine deaths prior to 20 weeks of pregnancy). ${ }^{4}$ However both Swedish studies identified an increased risk for neonatal death if women required non-obstetric surgery. Mazze's study identified a relative risk (RR) of 2.1 [95\% confidence interval (CI) 1.6-2.7] for neonatal death within seven days of delivery amongst women having non-obstetric surgery. Reedy's study did not identify an increased risk for neonatal death during the first week of delivery, but an increased risk of death 29 to 365 days from delivery (RR 2.0; 95\% CI 0.9, 4.7). In addition, both studies identified an increased risk of intra-uterine growth-restriction following non-obstetric surgery. Unfortunately, the studies did not adjust for confounding variables beyond age and parity of the mother, delivery unit and year of birth. Presumably women requiring surgery during pregnancy were dif-

From the Department of Anaesthesia, Mount Sinai Hospital, University of Toronto, Toronto, Ontario, Canada.

Address correspondence to: Dr. Alison Macarthur, Department of Anaesthesia, Mount Sinai Hospital, 600 University Avenue, Toronto, Ontario M5G 1X5, Canada. Phone: 416-586-5270; Fax: 416-586-8664; E-mail: Alison.Macarthur@uhn.on.ca 
ferent from women not requiring surgery, but it appears that non-obstetric surgery is associated with deleterious conditions for fetal development. We are at a loss as to when the risk to the fetus occurs, intraoperatively or in association with the duration of the disease and recovery.

I head into murky waters when evaluating the evidence for fetal heart monitoring during non-obstetric surgery. Most respected obstetric anesthesia texts and teachers will advocate FHR monitoring during nonobstetric surgery once the fetus has reached a gestational age of viability. ${ }^{6,7}$ Unfortunately, this advice is founded on the use of FHR variability as a measure of intrapartum fetal well-being from 25 weeks gestation forward, ${ }^{8,9}$ and possibly from medico-legal concerns of liability if FHR monitor is not used and an adverse outcome occurs. Evidence from studies evaluating the FHR monitoring during non-obstetric surgery is based only upon case reports ${ }^{10}$ and a case series of ten patients. ${ }^{11}$ While most fetuses survived, there were frequent events of intraoperative FHR abnormalities and uterine contractions requiring intervention. National organizations have been unable to establish clear guidelines as evidenced by the most recent opinion from the American College of Obstetricians and Gynecologists, which states, "there are no data that allow us to make specific recommendations". ${ }^{12}$ Instead the committee opinion recommends that individualized assessments be made based upon the "gestational age, type of surgery and facilities available". This statement implies that surgery may be required at facilities that cannot provide obstetric care, whether it is due to an urgent surgical event that precludes transfer or to technically challenging cases only operable in few centres. Clearly the decision requires input of all specialty caregivers (neonatalogy, maternal-fetal medicine obstetricians, surgeons, anesthesiologists) and parents.

Back to our case, a fetus of 28 weeks, of unidentified sex and of presumably normal weight for gestational age. After the 25th gestational week and over $800 \mathrm{~g}$, most neonates have a predicted mortality rate of less than $25 \%$ and even lower if female. ${ }^{13}$ Therefore, this fetus was beyond the threshold of viability and required consideration for fetal surveillance. The duration of the surgical procedure and general anesthetic, and the potential for vasoactive agent use during surgery would have weighed towards using FHR monitoring. Against this would have been practical issues such as providing qualified individuals to interpret the FHR tracing and individuals capable of providing the ultimate intervention, an operative delivery. In the end, the collective decision was made to not monitor the fetus, but provide stable maternal hemo- dynamics, oxygenation and ventilation. The final outcome was successful for both mother and baby. However, we may have a false sense of security in providing the best care for the fetus when we state that the "baby is best looked after when the mother's health is optimized". A recent case report supports this concern as intraoperative fetal bradycardia occurred, requiring urgent Cesarean delivery despite textbook optimization of maternal health. ${ }^{14}$ But anecdotal evidence is the least valuable category of evidence, insufficient to guide future decisions on fetal monitoring use. We require large sample evidence of FHR patterns during non-obstetric surgery. Our respective anesthetic and obstetric societies would do well in supporting prospective studies on fetal surveillance during non-obstetric surgery. Until then, the individual caregivers must await the conclusion of the pregnancy to decide if their decision was correct.

\section{Craniotomie pour méningiome suprasel- laire pendant la grossesse : le rôle du monitorage foetal}

L'étude, présentée dans le Journal canadien d'anesthésie, de l'apparition brutale d'une affection neurologique chez une parturiente à 28 semaines de grossesse montre la difficulté de traiter une atteinte non obstétricale pendant la grossesse. ${ }^{1}$ Dans ce cas, une femme ayant les symptômes neurologiques graves d'un méningiome suprasellaire doit subir un traitement chirurgical d'urgence avant que le fotus ait atteint un âge gestionnel raisonnable permettant une naissance viable. La chirurgie non obstétricale pendant la grossesse n'est pas nouvelle et a été réalisée selon diverses techniques, comme la laparoscopie et la circulation extracorporelle. Les risques de cette chirurgie comprennent généralement des conséquences tératogènes et la survenue prématurée du travail et de l'accouchement. La probabilité d'un travail et d'un accouchement prématurés après une intervention neurochirurgicale est plus faible que dans le cas d'une intervention intra-abdominale et il n'y a habituellement plus de risque de tératogénicité après la dou- 
zième semaine. Cependant, une asphyxie fotale peropératoire demeure toujours possible ainsi que le risque de ne pouvoir la détecter par le monitorage peropératoire externe de la fréquence cardiaque foetale (FCF). Un examen de la documentation médicale permet de définir ce risque et d'évaluer l'utilité du monitorage de la FCF.

Si les foetus étaient à risque élevé d'asphyxie peropératoire, les grossesses qui se poursuivent après une intervention chirurgicale non obstétricale présenteraient des taux accrus de mort intra-utérine (avortement, mort-né), de mort néonatale, de retard de croissance intra-utérine ou de déficit neurologique sévère. Pour montrer l'augmentation des taux, il faudrait comparer des parturientes qui ont subi une intervention non obstétricale pendant la grossesse à des parturientes qui n'ont pas été opérées. On sait que des complications, comme la mortalité ou la morbidité, sont évaluées plus efficacement par des études observationnelles, car ce serait contraire à l'éthique de réaliser l'essai clinique randomisé de référence. Ce qui supposerait une répartition aléatoire des femmes enceintes afin de subir une intervention chirurgicale réelle ou simulée. D'une part, une étude comparative de cohortes commencerait avec la population de toutes les femmes enceintes et comparerait ensuite la situation des femmes qui ont subi, ou non, une intervention chirurgicale non obstétricale. Une étude castémoins devrait, d'autre part, désigner d'abord les femmes qui ont présenté des complications (mort-né ou mort néonatale) et les comparer aux femmes qui n'en ont pas présenté, tout en recherchant les facteurs de risque potentiels comme les interventions chirurgicales non obstétricales.

Parmi les études de cohortes publiées, nous avons repéré quatre chercheurs de Suède, du Canada et des États-Unis qui ont utilisé des données représentatives sur l'évolution des naissances. ${ }^{2-5}$ Les quatre études comprennent environ 2000000 de Suédoises enceintes sur 20 ans, 100000 Canadiennes sur sept ans et 70000 Américaines sur dix ans. L'incidence de chirurgie non obstétricale était de $0,2 \%$ à $0,79 \%$. Les deux études suédoises ont comparé les taux de mortsnés, de morts néonatales et de naissances prématurées entre les femmes qui ont subi, ou non, une opération non obstétricale.,3 Aucune étude n'a montré de risque accru de mortinaissance après ce type d'opération. L'étude canadienne n'a pas non plus isolé de risque supplémentaire d'avortement (mort intrautérine avant 20 semaines de grossesse).${ }^{4}$ Cependant, les deux études suédoises ont présenté une hausse du risque de mort néonatale chez les femmes qui avaient dû subir une opération non obstétricale. L'étude de
Mazze a montré un risque relatif (RR) de 2,1 [intervalle de confiance (IC) de $95 \%, 1,6-2,7]$ de mort néonatale pendant les sept jours qui suivent l'accouchement de femmes qui ont eu une opération non obstétricale. L'étude de Reedy n'a pas montré de risque accru de mort néonatale pendant la première semaine, mais un risque plus élevé de décès de 29 à 365 jours après la naissance (RR de 2,0 ; IC de $95 \%$, $0,9,4,7)$. De plus, les deux études montrent un risque accru de retard de croissance intra-utérine à la suite d'une intervention chirurgicale non obstétricale. Malheureusement, les études n'ont pas été ajustées pour des variables confondantes autres que l'âge et le nombre de grossesses de chaque mère, le lieu et l'année des accouchements. Les femmes opérées pendant la grossesse sont probablement différentes des femmes non opérées, mais on a trouvé que la chirurgie non obstétricale nuit au développement foetal. Or, nous ne savons toujours pas quand survient le risque pour le foetus, pendant l'opération ou selon la durée de l'affection et de la récupération.

Il est très difficile d'évaluer clairement l'évidence du monitorage du cour foetal pendant une opération non obstétricale. La plupart des publications et des professeurs d'anesthésie respectés conseillent le monitorage de la FCF en chirurgie non obstétricale dès que le foetus a atteint un âge gestionnel de viabilité. ${ }^{6,7}$ Malheureusement, ce conseil est fondé sur l'usage de la variabilité de la FCF comme mesure de l'état foetal intra-partum à partir de 25 semaine de grossesse, ${ }^{8,9}$ et possiblement sur des préoccupations médico-légales de responsabilité si le moniteur de FCF n'est pas utilisé et que des complications surviennent. Seules les études de cas $^{10}$ et une petite série de dix patients ${ }^{11}$ fournissent des données sur l'évaluation du monitorage de la FCF pendant des interventions non obstétricales. Même si la majorité des foetus survivent, on relève de fréquentes anomalies peropératoires de la FCF et des contractions utérines qui nécessitent une intervention. Les organismes internationaux n'ont pu établir de directives claires comme le montre la plus récente déclaration de l'American College of Obstetricians and Gynecologists disant "qu'aucune donnée ne permet de faire des recommandations spécifiques». ${ }^{12}$ L'avis du comité est plutôt de recommander des évaluations individualisées fondées sur «l'âge gestationnel, le type de chirurgie et les aménagements disponibles». Cette déclaration implique que la chirurgie puisse être nécessaire dans un milieu qui ne fournit pas de soins obstétriques, que ce soit requis comme une urgence chirurgicale excluant le transfert de la patiente ou comme un cas techniquement complexe opérable dans certains centres seulement. La 
décision doit clairement reposer sur les suggestions des parents et des soignants de toutes les spécialités : néonatalogie ainsi qu'obstétriciens, chirurgiens et anesthésiologistes de médecine materno-fœtale.

Revenons au cas du foetus de 28 semaines, de sexe non identifié et de poids probablement normal pour l'âge gestationnel. Après la $25^{\mathrm{e}}$ semaine de grossesse et pour un poids de plus de $800 \mathrm{~g}$, la plupart des nouveau-nés présentent un taux de mortalité de moins de $25 \%$ et encore moins chez les filles. ${ }^{13}$ En conséquence, ce foetus se situait au delà du seuil de viabilité, ce qui commandait une surveillance fotale. La durée de l'intervention chirurgicale et de l'utilisation de l'anesthésique générale, de même que l'usage possible d'agent vasoactif pendant l'opération auraient dû inciter à utiliser le monitorage de la FCF. Par contre, il fallait considérer certaines questions pratiques comme la présence de personnel qualifié pour interpréter les tracés de la FCF et d'individus aptes à faire une césarienne le cas échéant. Finalement, la décision collective a été prise de ne pas placer le foetus sous monitorage, mais d'assurer la stabilité hémodynamique, l'oxygénation et la ventilation de la mère. L'issue finale a été heureuse pour la mère et l'enfant. Toutefois, nous pourrions avoir un faux sentiment de sécurité quand nous affirmons que «le bébé est mieux protégé lorsque la santé de la mère est optimale». Une récente étude de cas fait état de cette situation alors qu'une bradycardie foetale peropératoire est survenue et a nécessité une césarienne d'urgence malgré l'optimisation classique de la santé maternelle. ${ }^{14}$ Mais les évidences anecdotiques représentent la catégorie de preuves la moins valable, insuffisantes pour guider de futures décisions sur l'usage du monitorage fotal. Nous avons besoin d'évidences claires découlant du monitorage de grands échantillons de FCF pendant la chirurgie non obstétricale. Nos associations professionnelles respectives, en anesthésie et en obstétrique, gagneraient à appuyer des études prospectives sur la surveillance foetale pendant la chirurgie non obstétricale. D'ici là, les soignants doivent attendre la fin de la grossesse pour savoir si leur décision a été la bonne.

\section{References}

1 Balki M, Manninen PH. Craniotomy for suprasellar meningioma in a 28 -week pregnant woman without fetal heart rate monitoring. Can J Anesth 2004; 51: 573-76.

2 Mazze RI, Kallen B. Reproductive outcome after anesthesia and operation during pregnancy: a registry study of 5404 cases. Obstet Gynecol 1989; 161: 1178-85.

3 Reedy MB, Kallen B, Kuehl TJ. Laparoscopy during pregnancy: a study of five fetal outcome parameteres with use of the Swedish Health Registry. Am J Obstet Gynecol 1997; 177: 673-9.

4 Duncan PG, Pope WD, Cohen M, Greer N. Fetal risk of anesthesia and surgery during pregnancy.

Anesthesiology 1986; 64: 790-4.

5 Jenkins TM, Mackey SF, Benzoni FM, Tolosa JE, Sciscione $A C$. Non-obstetric surgery during gestation: risk factors for lower bithweight. Aust N Z J Obstet Gynaecol 2003; 43: 27-31.

6 Cohen SE. Nonobstetric surgery during pregnancy. In: Chestnut DH (Ed.). Obstetric Anesthesia. New York: Mosby; 1999: 279-302.

7 Hawkins JL. Anesthesia for the pregnant patient undergoing nonobstetric surgery. ASA 2003 annual meeting refresher course lecture, San Francisco.

8 Burrus DR, O'Shea TM Jr, Veille JC, Mueller-Henbach $E$. The predictive value of intrapartum fetal heart rate abnormalities in the extremely premature infant. Am J Obstet Gynecol 1994; 171: 1128-32.

9 Baird SM, Ruth DJ. Electronic fetal monitoring of the preterm fetus. J Perinat Neonatal Nurs 2002; 16: 12-24.

10 Kendrick JM, Neiger R. Intraoperative fetal monitoring during nonobstetric surgery (Letter). J Perinatol 2000; 4: 276-8.

11 Kendrick JM. Fetal uterine response during maternal surgery. Am J Matern Child Nurs 1994; 19: 165-70.

12 Anonymous. Nonobstetric surgery in pregnancy. ACOG Committee Opinion No. 284. Obstet Gynecol 2003; 102: 431 .

13 Anonymous. Perinatal care at the threshold of viability. ACOG Practice Bulletin No. 38. Obstet Gynecol 2002; 100: 617-24.

14 Ong BY, Baron K, Stearns EL, Baron C, Paetkau D, Segstro $R$. Severe fetal bradycardia in a pregnant surgical patient despite normal oxygenation and blood pressure. Can J Anesth 2003; 50: 922-5. 